\title{
A Comparison of the Clinical Features and Intraoperative Findings in Cholesteatoma Patients with and without Sinus Tympani Invasion
}

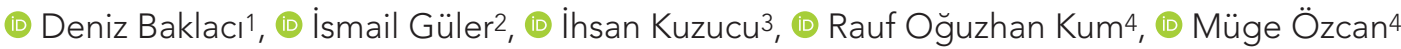 \\ ${ }^{1}$ Bülent Ecevit University Faculty of Medicine, Department of Otolaryngology, Zonguldak, Turkey \\ ${ }^{2}$ Ankara Medipol University Faculty of Medicine, Department of Otolaryngology, Ankara, Turkey \\ ${ }^{3}$ Medisun Private Hospital, Clinic of Otolaryngology, Ankara, Turkey \\ ${ }^{4}$ Ankara City Hospital, Clinic of Otolaryngology, Ankara, Turkey
}

Cite this article as: Baklacı D, Güler İ, Kuzucu I, Kum RO, Özcan M. A Comparison of the Clinical Features and Intraoperative Findings in Cholesteatoma Patients with and without Sinus Tympani Invasion. J Acad Res Med 2020;10(2):138-42

\begin{abstract}
Objective: The sinus tympani (ST) comprise one of the most hidden areas in the human body. It is one of the most common locations of residual cholesteatomas and is in close proximity with the facial nerve and stapes. These characteristics render ST as a key factor in chronic otitis media surgeries. This study aimed to investigate the clinical features and intraoperative findings of cholesteatoma patients with and without ST invasion (STI).

Methods: One hundred and fifty-one cholesteatoma patients who had undergone the canal wall-down procedure at our center were retrospectively reviewed. They were categorized into two groups: cholesteatoma patients with and without STI. Comparisons were made between the two groups in terms of the disease duration, surgical technique, rate of facial canal dehiscence (FCD), and number of locations of FCD and erosion of the stapes suprastructure. The mean hearing gain of the patients who underwent hearing reconstruction was compared between both groups.

Results: The rates of disease duration $>5$ years, radical mastoidectomy surgery, and erosion of the stapes suprastructure were significantly higher in patients with STI than in those without STI. Mean hearing gain was significantly higher in patients without STI than in those with STI. The numbers of locations and rate of FCD were also significantly higher in patients with STI than in those without STI.

Conclusion: The presence of STI in cholesteatoma patients is a significant intraoperative finding for the predicting the extent of FCD. STI should serve as a warning to surgeons because it indicates a potential for less functional outcomes due to erosion of the stapes suprastructure.

Keywords: Sinus tympani, cholesteatoma, facial canal, stapes suprastructure
\end{abstract}

\section{INTRODUCTION}

The sinus tympani (ST) are one of the most obscure areas in the human body. It lies medial to the pyramidal eminence, stapedial muscle, and facial nerve and lateral to the vestibule and posterior semicircular canal (1). The ST is clinically important during chronic otitis media surgery because of the risk of cholesteatoma recurrence due to the incomplete removal of the growth from the ST and conductive hearing loss due to its close proximity with the oval window and stapes suprastructure $(2,3)$. The cholesteatoma

ORCID IDs of the authors: D.B. 0000-0001-8449-4965; I.G. 0000-0001-6093-6757; I.K. 0000-0001-5773-4126; R.O.K. 0000-0002-9639-0204; M.Ö.

0000-0003-2384-3564 
located at the ST may come in contact with the stapes for a longer period with a higher pressure and may lead to erosion of the stapes suprastructure and less favorable functional outcomes.

The close proximity of the ST with the facial nerve makes the nerve more vulnerable during cholesteatoma surgery, especially if dehiscence is present in the bone covering the nerve (4-14). The facial canal dehiscence (FCD) may be caused by developmental bony defects or bony erosion caused by the enzymatic or pressure effect of cholesteatoma located at the ST. An unobserved FCD in the vicinity of the ST may be a risk factor for iatrogenic facial paralysis during the removal of cholesteatoma from the ST.

In this study, we aimed to compare the clinical features and intraoperative findings in cholesteatoma patients with and without ST invasion (STI).

\section{METHODS}

The study protocol was approved by the local ethical committee (approval number: E-18-1859, date: 11 April, 2018). Overall, 151 cholesteatoma patients ( 85 males, 66 females; $15-80$ years of age; average age 42.9 years) who had undergone the canal wall-down procedure (CWDP) [51 radical mastoidectomy (RM), 100 modified radical mastoidectomy (MRM)] at our tertiary referral center between January 2010 and December 2017 were retrospectively reviewed. All the patients underwent otoscopic examination and audiometric investigation. Preoperative imaging of the temporal bone was obtained for all the patients and included highresolution computed tomography (HRCT) or magnetic resonance imaging. The diagnosis of cholesteatoma was confirmed by histopathological examination. The disease duration, type of surgeries, hearing gain, and operation records, including information about the facial canal, STI, and stapes integrity, were documented for all the patients. The patients were categorized into two groups: cholesteatoma with STI and cholesteatoma without STI.

Our main indicators for CWDP were extensive cholesteatoma advancing into the mastoid and beyond, eustachian tube (anteromedial to the ossicles) or ST, extensive damage of the external auditory canal by disease, failure of previous canal wall-up surgery with recurrent cholesteatoma from epitympanic retraction pockets, patients with poor preoperative auditory thresholds, complicated cases, and the patients whose postoperative followup constitutes a problem. Patients who had undergone a revision tympanomastoidectomy and those with aural or intracranial complications were excluded from the study.

\section{Intraoperative Findings}

FCD observations were made with an operating microscope and confirmed by palpation with a blunt pick. We used the Moody and Lambert (13) classification for describing the FCD location. FCD located at the tympanic segment (TS) of the nerve was divided into three groups: [pure tympanic (PT); directly superior to the oval window], [geniculate ganglion (GG); proximal to the cochleariform process], and [mastoid genu (MG); distal to the oval window]. Combinations of PT, GG, and MG were also calculated. Dehiscence occurring in the mastoid segment of the nerve was noted as mastoid.

To evaluate the extent of cholesteatoma, the posterior canal wall was lowered to the level of the facial ridge. In patients where the cholesteatoma extended into the ST, the cholesteatoma was removed from the ST with a blunt pick and cottonoid.

\section{Ossicular Reconstruction in CWDP}

Ossicular chain reconstruction was performed in the presence of an intact and/or mobile stapes and good cochlear function. The integrity of the stapes suprastructure was assessed by the presence or absence of stapes footplates. Footplate mobility was evaluated by eliciting the round window reflex. Temporalis fascia graft was placed under the remnant of the anterior tympanic membrane and over the enlarged inferior canal wall. A small piece of cartilage (conchal) was placed between the fascia graft and stapes head. If the stapes suprastructure was absent and footplate mobile, we used titanium ossicular replacement prosthesis with a cartilage cap and place it between the footplate and temporalis fascia graft.

All the procedures performed were in accordance with the ethical standards of our institution and with the 1964 Helsinki declaration and its later amendments or comparable ethical standards. Written informed consent was obtained from all individual participants prior to surgery.

All the patients underwent preoperative and postoperative (at 6 months) pure-tone audiometry measurements at 0.5, 1, 2, and $4 \mathrm{kHz}$ and at $3 \mathrm{kHz}$ frequencies using an Interacoustic AC40 (Middelfart, Denmark) clinical audiometer. The average of the values at 2 and $4 \mathrm{kHz}$ was used to calculate the value for 3 $\mathrm{kHz}$. Preoperative and postoperative air and bone conduction thresholds were measured at these four frequencies. The air-bone gap (ABG) was calculated as the average difference between the air and bone conductions at four frequencies $(0.5,1,2$, and $3 \mathrm{kHz})$.

\section{Statistical Analysis}

The SPSS statistical software (SPSS 21.0 for Windows, Inc., Chicago, IL, USA) was used for data analysis. Quantitative data were presented as mean \pm standard deviation or median and interquartile range and categorical variables were presented as percentages. The data was tested for normal distribution using the Kolmogorov-Smirnov test. Student's t-test or Mann-Whitney U tests, as appropriate, were used to compare continuous variables. Chi-square test was used to identify statistically significant differences between categorical variables. A 2-tailed $p<0.05$ was considered significant.

\section{Results}

A total of 151 patients (mean age: $36.2 \pm 13.2$ years) with 151 operated ears (80 left, 71 right) who met the aforementioned criteria were evaluated. The overall STI rate was $41.7 \%$ (63 patients). 
The disease duration was $\square 5$ years in 31 patients (20.5\%) and $\square 5$ years in the remaining 120 patients (79.5\%). STI was observed in 57 of 120 patients (47.5\%) with a disease duration $\square 5$ years and in 6 of 31 patients (19.4\%) with a disease duration $\square 5$ years. The rate of the disease duration $\square 5$ years was significantly higher in patients with STI than in those without STI ( $p=0.005$, Table 1).

Overall, 36 of 63 patients (57.1\%) with STI and 15 of 88 patients (17\%) without STI had undergone RM and this difference was statistically significant ( $p<0.001$, Table 2).

The mean preoperative $A B G$ was $33.3 \pm 5.2 \mathrm{~dB}$ in patients with $\mathrm{STI}$ and $29.2 \pm 6.1 \mathrm{~dB}$ in those without STI. There was a statistically significant difference between the groups in terms of preoperative ABG $(p<0.001)$. The mean postoperative $A B G$ was $30.8 \pm 5.9 \mathrm{~dB}$ in patients with STI and $22.6 \pm 5.1 \mathrm{~dB}$ in patients without STI. There was a statistically significant difference between the groups in terms of postoperative ABG $(p<0.001)$. The mean hearing gain was $2.4 \pm 1.1 \mathrm{~dB}$ in patients with STI and $6.5 \pm 5.8 \mathrm{~dB}$ in those without STI and this difference was statistically significant $(p<0.001)$.

The overall FCD rate was $33.8 \%$ and FCD was observed in 34 of 63 patients (54\%) with STI and 17 of 88 patients (19.3\%) without STI. Among the 51 patients with FCD, dehiscence was located at the TS in 47 patients (92\%). The locations of dehiscence were as follows: GG, 2 patients (4.2\%); PT, 13 patients (27.6\%); MG, 4 patients (8.6\%); PT+GG, 9 patients (19.2\%); PT+MG, 10 patients (21.2\%); and GG+PT+MG, 9 patients (19.2\%). Overall, 4 patients (8\%) had both tympanic and mastoid segment dehiscence. There were at least 2 locations of FCD in 31 of 34 patients (96.9\%) with STI and 1 of 17 patients (6.2\%) without STI (Figure 1). There was a statistically significant difference between the groups in terms of the number of FCD location ( $p<0.001$, Table 3).

Erosion of the stapes suprastructure was observed in 46 of 63 patients (73\%) with STI and in 37 of 88 patients (42\%) without STI. There was a statistically significant difference between both groups in terms of erosion of the stapes suprastructure $(p<0.001$, Table 3).

\section{Discussion}

The ST has always been considered as important during cholesteatoma surgery due to its inability to be directly visualized under microscopes and because it is the most common location of residual cholesteatomas $(2,3,15,16)$. The ST also has a critical anatomical proximity with the facial canal, and this proximity obstructs its surgical manipulation, especially when there is an anatomical defect or variation in the canal. The specificity

\begin{tabular}{|c|c|c|c|c|}
\hline Disease duration & $\begin{array}{l}\text { STI (+) } \\
(n=63)\end{array}$ & $\begin{array}{l}\text { STI (-) } \\
(n=88)\end{array}$ & $\begin{array}{l}\text { Total } \\
(n=151)\end{array}$ & $\mathrm{p}$ \\
\hline Less than 5 years & $6(9 \%)$ & $25(28 \%)$ & $31(20 \%)$ & \multirow{2}{*}{$0.005^{\star}$} \\
\hline More than 5 years & $57(91 \%)$ & $63(72 \%)$ & $120(80 \%)$ & \\
\hline \multicolumn{5}{|c|}{ N: Number of patients, STI: sinus tympani invasion, ${ }^{*} p<0.05$} \\
\hline
\end{tabular}

and sensitivity of imaging systems are low for the detection of perioperative FCD (17). Therefore, ear surgeons should be familiar with the pathologies having a high likelihood of observing FCD, especially in cholesteatoma patients. Sometimes, surgeons may reasonably approach the facial nerve path more carefully and even risk the incomplete removal of the cholesteatoma when FCD is intraoperatively observed. FCD had been previously demonstrated to negatively affect surgical outcomes, including suboptimal hearing results and a potential requirement for revision surgery (18).

In our study, 63 of 151 patients (41.7\%) had cholesteatoma invading into the ST. The disease duration was $\square 5$ years in 57 of 63 patients (90.4\%) with STI and in 63 of 88 patients (70.1\%) without STI. A longer disease duration prior to the surgery may provide insight into the extent of the disease in the ST. Overall, 36 of 63 (57\%) with STI and 15 of 88 (17\%) without STI had undergone RM. These results indicated that the extent of cholesteatoma into the ST affects a surgeon's choice for RM $(p<0.001)$. The assessment of

Table 2. Distribution of the type of surgery according to STI

\begin{tabular}{|l|l|l|l|l|}
\hline Type of surgery & $\begin{array}{l}\text { STI (+) } \\
(\mathbf{n = 6 3 )}\end{array}$ & $\begin{array}{l}\text { STI (-) } \\
(\mathbf{n = 8 8 )}\end{array}$ & $\begin{array}{l}\text { Total } \\
(\mathbf{n = 1 5 1 )}\end{array}$ & $\mathbf{p}$ \\
\hline Radical & 36 & 15 & 51 & \\
mastoidectomy & $(57.1 \%)$ & $(17 \%)$ & $(33.7 \%)$ & $<0.001{ }^{*}$ \\
\hline $\begin{array}{l}\text { Modified radical } \\
\text { mastoidectomy }\end{array}$ & 27 & 73 & 100 & \\
\hline n: Number of patients, STI: & $(42.9 \%)$ & $(83 \%)$ & $(66.3 \%)$ & \\
\hline
\end{tabular}

\begin{tabular}{|c|c|c|c|c|}
\hline & $\begin{array}{l}\text { STI (+) } \\
(n=63)\end{array}$ & $\begin{array}{l}\text { STI (-) } \\
(n=88)\end{array}$ & $\begin{array}{l}\text { Total } \\
(n=151)\end{array}$ & $p$ \\
\hline Facial canal dehiscence & $\begin{array}{l}34 \\
(54 \%)\end{array}$ & $\begin{array}{l}17 \\
(19.3 \%)\end{array}$ & $\begin{array}{l}51 \\
(33.8 \%)\end{array}$ & $<0.001^{*}$ \\
\hline One location & $\begin{array}{l}3 \\
(3.1 \%)\end{array}$ & $\begin{array}{l}16 \\
(93.8 \%)\end{array}$ & $\begin{array}{l}19 \\
(37 \%)\end{array}$ & \multirow{2}{*}{$<0.001$ * } \\
\hline More than one location & $\begin{array}{l}31 \\
(96.9 \%)\end{array}$ & $\begin{array}{l}1 \\
(6.2 \%)\end{array}$ & $\begin{array}{l}32 \\
(63 \%)\end{array}$ & \\
\hline $\begin{array}{l}\text { Stape suprastructure } \\
\text { erosion }\end{array}$ & $\begin{array}{l}46 \\
(73 \%)\end{array}$ & $\begin{array}{l}37 \\
(42 \%)\end{array}$ & $\begin{array}{l}83 \\
(55 \%)\end{array}$ & $<0.001^{*}$ \\
\hline
\end{tabular}

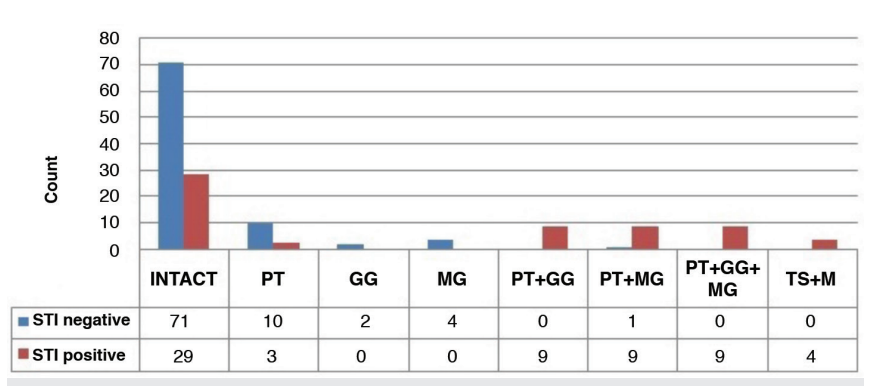

Figure 1. Status of the facial canal and sites of facial canal dehiscence with respect to STI

STI: Sinus tympani invasion, PT: pure tympanic, GG: geniculate ganglion, MG: mastoid genu, TS: tympanic segment, M: mastoid 
ossicular chain defects could explain the tendency of performing RM in patients with STI. We assessed the integrity of the stapes suprastructure according to the proximity of the oval window and stapes with ST. Our results demonstrated that the stapes suprastructure was eroded in 46 patients (73\%) with STI and in 37 patients (42\%) without STI. The close proximity of the ST with the stapes may cause the cholesteatoma to come in contact with the stapes for a longer period and with a higher pressure. The absence of the stapes suprastructure may complicate ossicular reconstruction.

Postoperative hearing outcomes were found to be better in cholesteatoma patients with the presence of the stapes suprastructure (19). Hence, STI resulting from cholesteatoma should alert surgeons regrading potentially less functional outcomes, and the preoperative evaluation of STI by HRCT may provide insights concerning the integrity of the stapes suprastructure.

FCD may be caused by developmental defects due to failure during the ossification process of the bony canal or bony erosion caused by cholesteatoma and inflammation (20). The mechanism of bony erosion resulting from cholesteatoma has indicated that bony erosion is due to the enzymatic or compression effect of cholesteatoma (21). The rate of dehiscence was reportedly higher (ranging between $55 \%$ and $72 \%$ ) in other anatomical studies (22-24). Yetiser et al. (10) have reported an FCD rate of $11 \%$ in non-cholesteatoma patients. Bayazit et al. (20) have reported an FCD rate of $8.9 \%$ in non-cholesteatoma patients and $18.4 \%$ in cholesteatoma patients, whereas Ozbek et al. (14) have reported this rate at $37.2 \%$ in cholesteatoma patients. In the series of Magliulo et al. (17), which comprised 336 patients who had undergone mastoidectomy for cholesteatoma, this rate was reported as $27.1 \%$. Selesnick and Lynn-Macrae (4) reported this rate at $33 \%$ during primary surgery and $35 \%$ during revision surgery. Moreover, Genc et al. (25) have reported this rate at $32.7 \%$ in cholesteatoma patients.

However, no healthy controls were analyzed in our study. The rate of FCD was $33.8 \%$, which is similar to the results of Genc et al. (25) (32.7\%) and Selesnick and Lynn-Macrae (4) (33\%). The likely cause for this high percentage was that most of the patients included in our study had delayed or extensive disease. Our clinical approach for extensive cholesteatoma is CWDP. By this approach, we could accurately remove pathological growth from the middle ear and evaluate the FCD. The FCD rate was found to be significantly higher (54\%) in patients with STI than in patients without STI, possibly due to a longer duration of contact of the cholesteatoma with the bony canal covering the facial nerve in the middle ear. Magliulo et al. (17) found that the risk for FCD was approximately 3.5 times more likely in patients with disease duration $\square 5$ years. These findings indicate that the longer pressure and enzymatic effects of the cholesteatoma mass on the bony canal results in a higher incidence of FCD or microdehiscence is enzymatically enlarged by cholesteatoma with time. This theory is supported by the fact that the disease duration was significantly longer in patients with STI than in those without STI.

Hence, we may conclude that the gross dehiscence of the facial canal may be due to prolonged contact with cholesteatoma or enlargement of a microdehiscence resulting from the disease. Conversely, it can be stated that cholesteatoma located at the ST may cause erosion in the adjacent bony structures as it expands. The pressure effect may increase when the cholesteatoma in the ST enlarges. Due to the pressure and enzymatic effects of cholesteatoma in this narrow space, the microdehiscence on the facial canal can be transformed into a macrodehiscence.

Previous studies have demonstrated that the most common location of dehiscence is the TS of the facial canal. Reportedly, FCD was also mainly observed in the tympanic region (92\%). When we divided the number of locations of the tympanic dehiscences into three groups, the most common sites of dehiscence were the PT, MG, and GG, respectively. However, only mastoid dehiscence was not observed. The bony canal surrounding the facial nerve in the TS is quite thin; therefore, pathologies in the middle ear, i.e., cholesteatoma, infections, and inflammation, may directly damage the facial canal in these locations. In patients with STI, dehiscence was found at more than one location in 31 patients (96.9\%). The number of dehiscence locations was correlated with STI. In patients with STI, dehiscence was observed at PT+GG in 9, $\mathrm{PT}+\mathrm{MG}$ in 9, PT+GG+MG in 9, and T+M in 4 patients. Hence, we may conclude that cholesteatomas invading the ST were more aggressive and extensive in terms of FCD, and a significant correlation was noted between STI and the extent of dehiscence. It should be kept in mind that the presence of dehiscence at one segment of the facial canal in patients with STI may indicate the presence of additional dehiscence at other regions of the canal.

\section{Study Limitations}

There are two limitations to be addressed in this study. Firstly, some personal biases may have crept in during the evaluation of the surgical findings. Secondly, in retrospective studies, some of the records obtained from medical charts may be incomplete or lost in the course of time, leading to missing data. Further prospective studies can provide more reliable and accurate data.

\section{CONCLUSION}

FCD may be a challenging issue during cholesteatoma surgery, even for experienced surgeons. Dehiscence is more common in patients when cholesteatoma invades the ST. Our study indicates that FCD is present in more than half of cholesteatoma patients with STI. Hence, for patients in whom STI is intraoperatively observed, their facial nerve is likely at risk. The presence of STI in cholesteatoma patients is a significant finding for the prediction and extent of FCD. Ear surgeons must cautiously evaluate the intraoperative findings that may suggest FCD, such as the presence of STI, especially in patients with extensive cholesteatoma. Hence, the prevention of facial injury should be a priority while operating upon cholesteatoma patients with 
STI. STI should serve as a warning to surgeons as it indicates a potential for less functional outcomes due to erosion of the stapes suprastructure in cholesteatoma patients.

Ethics Committee Approval: The study protocol was approved by the local ethical committee (approval number: E-18-1859, date: 11.04.2018).

Informed Consent: Written informed consent was obtained from all individual participants prior to surgery.

Peer-review: Externally peer-reviewed.

Author Contributions: Surgical and Medical Practices - D.B., I.G., I.K., R.O.K., M.Ö.; Concept - D.B., I.K., R.O.K., M.Ö.; Design - D.B., I.K., R.O.K., M.Ö.; Data Collection and/or Processing - D.B., I.G.; Analysis and/ or Interpretation - D.B., I.G., R.O.K., M.Ö.; Literature Search - D.B., I.G.; Writing Manuscript - D.B.

Conflict of Interest: The authors have no conflict of interest to declare.

Financial Disclosure: The authors declared that this study has received no financial support.

\section{REFERENCES}

1. Marchioni D, Mattioli F, Alicandri-Ciufelli M, Presutti L. Transcanal endoscopic approach to the sinus tympani: a clinical report. Otol Neurotol 2009; 30: 758-65

2. Weiss MH, Parisier SC, Han JC, Edelstein DR. Surgery for recurrent and residual cholesteatoma. Laryngoscope 1992; 102: 145-51.

3. Pulec J. Sinus tympani: retrofacial approach for the removal of cholesteatomas. Ear Nose Throat J 1996; 75: 86-8.

4. Selesnick SH, Lynn-Macrae AG. The incidence of facial nerve dehiscence at surgery for cholesteatoma. Otol Neurotol 2001; 22: 129-32.

5. Sheehy JL, Brackmann DE, Graham MD. Cholesteatoma surgery: residual and recurrent disease. A review of 1,024 cases. Ann Otol Rhinol Laryngol 1977; 86: 451-62.

6. Green JD Jr, Shelton C, Brackmann DE. latrogenic facial nerve injury during otologic surgery. Laryngoscope 1994; 104: 922-6.

7. Li D, Cao Y. Facial canal dehiscence: a report of 1,465 stapes operations. Ann Otol Rhinol Laryngol 1996; 105: 467-71.

8. Nilssen EL, Wormald PJ. Facial nerve palsy in mastoid surgery. J Laryngol Otol 1997; 111: 113-6.

9. Harvey SA, Fox MC. Relevant issues in revision canal-wall-down mastoidectomy. Otolaryngol Head Neck Surg 1999; 121: 18-22.
10. Yetiser S, Tosun F, Kazkayasi M. Facial nerve paralysis due to chronic otitis media. Otol Neurotol 2002; 23: 580-8.

11. Lin JC, Ho KY, Kuo WR, Wang LF, Chai CY, Tsai SM. Incidence of dehiscence of the facial nerve at surgery for middle ear cholesteatoma. Otolaryngol Head Neck Surg 2004; 131: 452-6.

12. Di Martino E, Sellhaus B, Haensel J, Schlegel JG, Westhofen M, Prescher A. Fallopian canal dehiscences: a survey of clinical and anatomical findings. Eur Arch Otorhinolaryngol 2005; 262: 120-6.

13. Moody MW, Lambert PR. Incidence of dehiscence of the facial nerve in 416 cases of cholesteatoma. Otol Neurotol 2007; 28: 400-4.

14. Ozbek C, Tuna E, Ciftci O, Yazkan O, Ozdem C. Incidence of fallopian canal dehiscence at surgery for chronic otitis media. Eur Arch Otorhinolaryngol 2009; 266: 357-62.

15. Badr-el-Dine M. Surgery of Sinus Tympani Cholesteatoma: Endoscopic Necessity. J Int Adv Otol 2009; 5: 158-65.

16. Marchioni D, Valerini S, Mattioli F, Alicandri-Ciufelli M, Presutti L. Radiological assessment of the sinus tympani: temporal bone HRCT analyses and surgically related findings. Surg Radiol Anat 2015; 37: 38592.

17. Magliulo G, Colicchio MG, Appiani MC. Facial nerve dehiscence and cholesteatoma. Ann Otol Rhinol Laryngol 2011; 120: 261-7.

18. Ocak E, Beton S, Mulazimoglu S, Meco C. Does dehiscence of the facial nerve canal affect tympanoplasty results? J Craniofac Surg 2016; 27: 3746.

19. Iurato $\mathrm{S}$, Marioni G, Onofri M. Hearing results of ossiculoplasty in AustinKartush group A patients. Otol Neurotol 2001; 22 :140-4.

20. Bayazit YA, Ozer E, Kanlikama M. Gross dehiscence of the bone covering the facial nerve in the light of otological surgery. J Laryngol Otol 2002; $116: 800-3$

21. Uno Y, Saito R. Bone resorption in human cholesteatoma: morphological study with scanning electron microscopy. Ann Otol Rhinol Laryngol 1995; 104: 463-8.

22. Moreano EH, Paparella MM, Zelterman D, Goycoolea MV. Prevalence of facial canal dehiscence and of persistent stapedial artery in the human middle ear: a report of 1000 temporal bones. Laryngoscope 1994; 104: 309-20.

23. Baxter A. Dehiscence of the fallopian canal. An anatomical study. J Laryngol Otol 1971; 85: 587-94.

24. Nomiya S, Cureoglu S, Kariya S, Morita N, Nomiya R, Nishizaki K, et al. Histopathological incidence of facial canal dehiscence in otosclerosis. Eur Arch Otorhinolaryngol 2011; 268: 1267-71.

25. Genc S, Genc MG, Arslan IB, Selcuk A. Coexistence of scutum defect and facial canal dehiscence. Eur Arch Otorhinolaryngol 2014; 271: 701-5. 